\title{
Celebrating Reviewers: your contribution to development of the discipline
}

People who review papers for this Journal are vital to both the development of the discipline and quality assurance of the Journal. Reviewing, we appreciate, is an additional task. We thank all those who have reviewed for Primary Health Care Research and Development helping to ensure that our publication is of the highest standard. A full list of reviewers over the past year is published as a Supplementary file (online only) to this Editorial.

This is an exciting time for primary health care research and for the dissemination of that research through journal publications with the recognition by Thomson Reuters ISI in their indexing of journals of the new category of Primary Health Care. Professor Chris van Weel, immediate past president of Wonca and a member of the Editorial Board of this Journal, has been instrumental in gaining this new category, which will raise the profile of this group of journals in the academic community (Noticeboard, 2011). This recognition of the discipline is a significant step in widening the understanding of the contribution of primary care and primary health care research to health and health care. Reeve and Mallen (2012) in this issue provide a commentary on Watts (2011: 5) paper: Blue Sky Research for Primary Care. In that paper Watts draws an analogy between the position of psychiatric research in the 1950s when he comments such research was '...poorly developed, and thought by some to be "unresearchable", This view obviously has the potential to stimulate debate such as that promoted by Reeve and Mallen but it does point to considerable concern within the primary care/primary health care research community about the position and recognition of the value of research in these areas. It is therefore vital to development of the discipline that there is timely publication of the genesis, process, outcomes and implementation of research findings to inform the research community, practitioners and the health care of the population.

Timely publication is dependent on the voluntary contribution of reviewers and Primary Health Care Research and Development has been very fortunate in the quality and responsiveness of reviewers to

(C) Cambridge University Press 2012 our requests. In undertaking reviews, the reviewer is bringing their expertise to the paper and applying and questioning the research or implementation process reported in the paper. These developments should help reviewers benchmark their own reviews. Importantly, the overall quality of a journal is determined by and dependent on the peer review process. Reviewers that inform the editors through their expertise of the strengths and weaknesses of research and development papers, make an enormous contribution to the editors' decision on the paper. Lee et al. (2002) demonstrated that citation rates are one of the most powerful predictors of research quality. The highest quality papers will eventually be cited most frequently by other authors and those citations are what will enable the journal to grow in stature and quality in the future. Thus, the scientific discipline of primary care research will also be enhanced, with the expectation that some of that research will eventually filter into primary health care practice.

In 2005 we held a workshop on the peer review process with Dr Jonathan Graffy and Dr David Crook at the SAPC Annual Scientific Meeting in Newcastle (Graffy et al., 2006). In the workshop, individuals commented on the value of undertaking reviews in developing their writing skills and in helping understand the publication process. By closely reviewing a paper, the reviewer has to engage with the requirements of a journal and this can help to inform their own future publications. People at the workshop commented that they would like to be able to see the reviews by the other reviewer(s) of a paper, again as a means to develop their own skills. Primary Health Care Research and Development has responded to this request and reviewers are now provided with copies of the other reviews on a paper and, additionally, with a copy of the letter sent to the author informing them of the outcome of the review process. Workshops, support from colleagues who are experienced reviewers and feedback on reviews are all ways in which reviewers can develop their reviewing skills. Recognition of the contribution of reviewing to the discipline was 
also important to the workshop participants and publication of the annual list of reviewers for this Journal is aimed at providing some of that recognition. Please let us know if there are other ways we can support and promote your role as a reviewer. If you are not yet a reviewer for the Journal, please go to the web site and register yourself as a reviewer: http://www.editorialmanager.com/phc/default.asp. If you are new to reviewing, perhaps you have a colleague who could mentor you; if you are an experienced reviewer, are there people in your department or setting who you could support to become reviewers?

In doing this, the reviewer is also developing and extending their own knowledge and in the process new ideas may be generated or new connections made. Such new ideas may then lead on to new research questions, research applications and findings. Thus, the process of reviewing contributes to the development of the discipline as discussed by Watts (2011).

\section{Ros Bryar and Sally Kendall Editors-in-Chief}

\section{Supplementary materials}

For supplementary material referred to in this article, please visit http://dx.doi.org/doi:10.1017/ S146342361200028X

\section{References}

Graffy, J., Bryar, R., Kendall, S. and Crook, D. 2006: Improving peer review. Primary Health Care Research and Development 7, 1-2.

Lee, K., Schotland, M., Bacchetti, P. and Bero, L. 2002: Association of journal quality indicators with methodological quality of clinical research articles. Journal of the American Medical Association 287, 2805-808.

Noticeboard. 2011: Primary health care journals impact factor. Retrieved 24 April 2012 from http://www.icgp.ie/go/library/ notice_board/99AA6425-19B9-E185-839D80EEAD3BADBE. html

Reeve, J. and Mallen, 2012: SAPC hot topic - capacity for Blue Sky Research in academic primary care. Primary Health Care Research and Development 13.

Watts, G. 2011: Blue Sky Research for Primary Care. A discussion paper. University of Glasgow, Glasgow. Retrieved 26 April 2012 from http://www.sapc.ac.uk/images/documents/blue.pdf 